\title{
Critical Impact Yaw for Long-Rod Penetrators
}

DOI:

$10.1115 / 1.4034620$

\section{Document Version}

Accepted author manuscript

Link to publication record in Manchester Research Explorer

\section{Citation for published version (APA):}

Kong, X., Li, Q. M., \& Fang, Q. (2016). Critical Impact Yaw for Long-Rod Penetrators. Journal of Applied Mechanics, Transactions ASME, 83(12), [121008]. https://doi.org/10.1115/1.4034620

\section{Published in:}

Journal of Applied Mechanics, Transactions ASME

\section{Citing this paper}

Please note that where the full-text provided on Manchester Research Explorer is the Author Accepted Manuscript or Proof version this may differ from the final Published version. If citing, it is advised that you check and use the publisher's definitive version.

\section{General rights}

Copyright and moral rights for the publications made accessible in the Research Explorer are retained by the authors and/or other copyright owners and it is a condition of accessing publications that users recognise and abide by the legal requirements associated with these rights.

\section{Takedown policy}

If you believe that this document breaches copyright please refer to the University of Manchester's Takedown Procedures [http://man.ac.uk/04Y6Bo] or contact uml.scholarlycommunications@manchester.ac.uk providing relevant details, so we can investigate your claim.

\section{OPEN ACCESS}




\title{
Critical impact yaw for long rod penetrators
}

\author{
Xiangzhen Kong ${ }^{+a}$, Q.M. Li ${ }^{\text {bc }}$, Qin Fang*a \\ ${ }^{a}$ State Key Laboratory for Disaster Prevention and Mitigation of Explosion and Impact, PLA University of Science and \\ Technology, Nanjing, Jiangsu Province, 210007, China \\ b School of Mechanical, Aerospace and Civil Engineering, Pariser Building, The University of Manchester, Sackville \\ Street, Manchester M13 9PL, UK \\ ${ }^{\mathrm{c}}$ State Key Laboratory of Explosion Science and Technology, Beijing Institute of Technology, Beijing 100081, China \\ * Corresponding authors: Q.M. Li: Qingming.Li@manchester.ac.uk; Qin Fang: fangqinjs@139.com \\ + First author: Xiangzhen Kong: ouckxz@163.com
}

\begin{abstract}
This paper presents an improved model for the critical impact yaw (or simply the critical yaw) in long rod penetration with considering the deceleration and rotation of the rod and the crater shape of the target. Two critical yaws, $\theta_{c 1}$ and $\theta_{c 2}$, under normal impact were identified, below which there is no contact between the rod and crater sidewall (for $\theta_{c 1}$ ) and between the rod and the crater entrance (for $\theta_{c 2}$ ) during the entire penetration process. Contact functions and iterative algorithms were proposed in order to obtain these two critical yaws numerically. The influences of four dominant non-dimensional numbers (i.e. the ratio of the target resistance to the rod strength $\lambda$, Johnson's damage number of the rod $\varsigma$, square root of the target-projectile density ratio $\mu$ and the diameter-length ratio of the rod $\psi$ ) on two critical yaws were studied for three typical rod-target systems (tungsten alloy rods penetrating steel targets, steel rods penetrating aluminum alloy targets, and steel rods penetrating steel targets). The relationship between two critical yaw angles was also discussed. A new empirical formula for the critical yaw $\theta_{c 2}$ was proposed based on the parametric Paper No. : JAM-16-1374. Corresponding authors: Li and Fang. Page 1
\end{abstract}


study results and dominant non-dimensional numbers, which extends the valid application range of the existing empirical formula.

Keywords: Critical yaw, long rod penetration, crater shape, empirical formula

\section{Introduction}

Extensive research has been done for the normal penetration of eroding long rods based on the Alekseevski and Tate (A-T) model proposed by Tate [1] and Alekseevskii [2] independently. However, impact yaw angle is unavoidable due to various causes, e.g. the sabot discard and the unsteadiness of flight orientation. Herein, the impact yaw is referred to the total initial yaw consisting of both pitch (up-down) and yaw (right-left), which was defined by Bjerke et al. [3] as the angle between the direction of the longitudinal axis of the rod and the centroid velocity vector of the rod. It will be simply termed as yaw in the present study unless otherwise stated. The centroid velocity vector of the rod is assumed to be normal to the target surface (i.e. zero oblique angle) without losing generality. Experimental observations showed the existence of a critical yaw, above which the depth of penetration (DOP) decreases rapidly with yaw [3, 4]. The critical yaw represents the start of contact between the rod body and the crater sideway [3]. If the initial yaw $\left(\theta_{0}\right)$ is smaller than the critical yaw $\left(\theta_{c}\right)$, the rod body will not collide with the crater sideway and the DOP of the rod is close to that in a corresponding normal penetration. However, if $\theta_{0}>\theta_{c}$, the collision between the rod body and crater sidewall may bend or break the rod, leading to a drastic reduction of rod's penetration capability.

There are limited investigations on the critical yaw for long rod penetration into infinite target. 
Based on the assumption that the instantaneous yaw angle does not change during penetration and that the rod does not erode, Bjerke et al. [3] suggested a geometrical method to determine the critical yaw, in which the crater diameter for the penetration of thick rolled homogeneous armor (RHA) targets by a tungsten alloy rod was given empirically as a function of impact velocity. Similar empirical formula was proposed by Walker et al. [5] with the same hypotheses. It should be noted that above formulae depend only on striking velocity and rod geometry without including the material properties of rod and target explicitly, which implies that they are only valid for the specific rod and target (i.e. tungsten alloys rod and RHA target) used in their studies [3, 5].

Yaziv et al. [6] applied A-T model to predict DOP of yawed rod in a steel target. They assumed that the erosion of the rod is related to its effective length (projected rod length in the velocity direction) while the dynamic equations of the rod are still related to its true length. The rotation of the uneroded part of the rod was not considered. This model is effective for low yaw angles. However, overestimation was observed when the yaw angle becomes large. Based on A-T model, Bukharev and Zhykov [7] established a yawed penetration model with the consideration of the transverse loads and the deceleration and rotation of the rod. However, the main purpose of Bukharev and Zhykov [7] is to take the initial yaw angle into account in the prediction of the depth of penetration. It is unable to predict the crater shape, and consequently, the critical yaw cannot be predicted because it requires the relative position of the rod to the crater sidewall. In addition, the transverse load in Ref. [7] was obtained based on the full contact between the rigid rod and the target, which did not agree with experimental observation [3]. Lee [8] proposed an engineering discrete model for yawed long rod. The yawed rod was assumed to be made of a series of continuous disk Paper No. : JAM-16-1374. Corresponding authors: Li and Fang. Page 3 
elements with finite length. Each element impacts the target to produce incremental DOP and change of crater shape. Therefore, the model is able to predict both the DOP and crater shape. However, the model assumed a constant penetration rate and ignored the rotation of the rod. A similar discrete model was applied by Rosenberg et al. [9], which focuses on the RHA targets and tungsten alloy rods and did not consider the rotation of the rod.

An improved model is proposed in the present paper for the critical yaw with the consideration of the deceleration and rotation of the rod and the crater shape change. The model, which is valid for low yaw angles, is introduced in Sec. 2 and is verified in Sec. 3 before it is used to study three typical cases in Sec. 4. Based on the predictions of the two critical yaws for three typical rod-target systems, a new empirical formula for $\theta_{c 2}$ is recommended and validated in Sec. 5, which is followed by conclusive remarks in Sec. 6.

\section{Model for critical yaw}

In this section, the governing equations of the motion of rod are presented. Then the method to determine the crater shape is described. Based on the relative position of the rod and crater, two critical yaws are obtained using an iterative algorithm when contact functions are introduced.

\subsection{Rod motion}

There are two main components of the rod motion during penetration at normal impact (zero oblique angle), i.e. the vertical translation and the rotation of the rod. The vertical penetration in the direction of the velocity vector is governed by the effective length $l_{e}$, as shown in Fig.1, i.e.

$$
\begin{gathered}
\frac{\rho_{p}}{2}(v-u)^{2}+Y_{p}=\frac{\rho_{t}}{2} u^{2}+R_{t} \\
l_{e}=(u-v)
\end{gathered}
$$




$$
\begin{gathered}
\dot{v}=-\frac{Y_{p}}{\rho_{p} l_{e}} \\
\dot{P}=u
\end{gathered}
$$

where superscript dot represents the derivative of time; $\rho_{p}$ and $\rho_{t}$ are the projectile and target densities, respectively; $v$ is the speed of the rear rigid portion of the rod; $u$ is the penetration speed; $Y_{p}$ is the strength of the projectile; $R_{t}$ is defined as the target resistance in the 1-D formulation; $l_{e}=l \cos \theta$ is the instantaneous effective rod length where $l$ is the true instantaneous rod length; $\theta$ refers to the instantaneous yaw angle; $d$ is the diameter of the rod; $P$ is the instantaneous DOP. Equations (1-4) reduce to A-T model when the yaw angle is zero. It should be noted that if the striking yaw is larger than the critical yaw, rod-target interaction will occur and transverse force should be introduced [10-11]. However, this issue is beyond the scope of this study.

If $R_{t} \geq Y_{p}$, the rod always behaves as fluid until penetration ceases (i.e. $u=0$ ) at $v=V_{c}=\sqrt{2\left(R_{t}-Y_{p}\right) / \rho_{p}}$. Then, the target will become a rigid body and the rod will deform plastically as what happens in a Taylor test [12], which will have no contribution to penetration. If $R_{t}<Y_{p}$, the rod behaves as fluid until $v$ drops to $v=V_{r}=\sqrt{2\left(Y_{p}-R_{t}\right) / \rho_{t}}$, from which, $v=u$ and the rod behaves as a rigid body. The present paper focuses only on the $R_{t} \geq Y_{p}$ case because the rigid body penetration stage for $R_{t}<Y_{p}$ in A-T model has some deficiencies, as pointed out in [13]. $R_{t}$ in A-T model (Eq.(1)) is usually treated as a constant. However, it has been noted that $R_{t}$ varies considerably during penetration [14], and therefore, it should be understood as an average target resistance during the penetration. Nevertheless, if the dependence of $R_{t}$ on penetration velocity can be specified, there is no difficulty to implement the velocity-dependent $R_{t}$ into the model.

The rotation of the undeformed rod about its instantaneous centroid is governed by Paper No. : JAM-16-1374. Corresponding authors: Li and Fang. Page 5 


$$
\ddot{\theta}=\frac{1}{I}\left(Y_{p} \frac{\pi d^{2}}{4 \cos \theta} \sin \theta \frac{l}{2}\right)=\frac{Y_{p} \tan \theta}{\rho_{p}\left(\frac{l_{e}^{2}}{6 \cos ^{2} \theta}+\frac{d^{2}}{8}\right)}
$$

where $\pi d^{2} /(4 \cos \theta)$ is the effective contact area of the rod's end surface with the target. $I=m\left(l^{2} / 12+d^{2} / 16\right)$ is the moment of inertia of the instantaneous rod about its centroid. One may note that Eqs. (1-5) are the same as those equations presented by Bukharev and Zhykov [7]. However, as mentioned previously, Bukharev and Zhykov [7]'s model did not consider the formation of crater, and therefore, can only be used to predict the depth of penetration, but not the critical yaw.

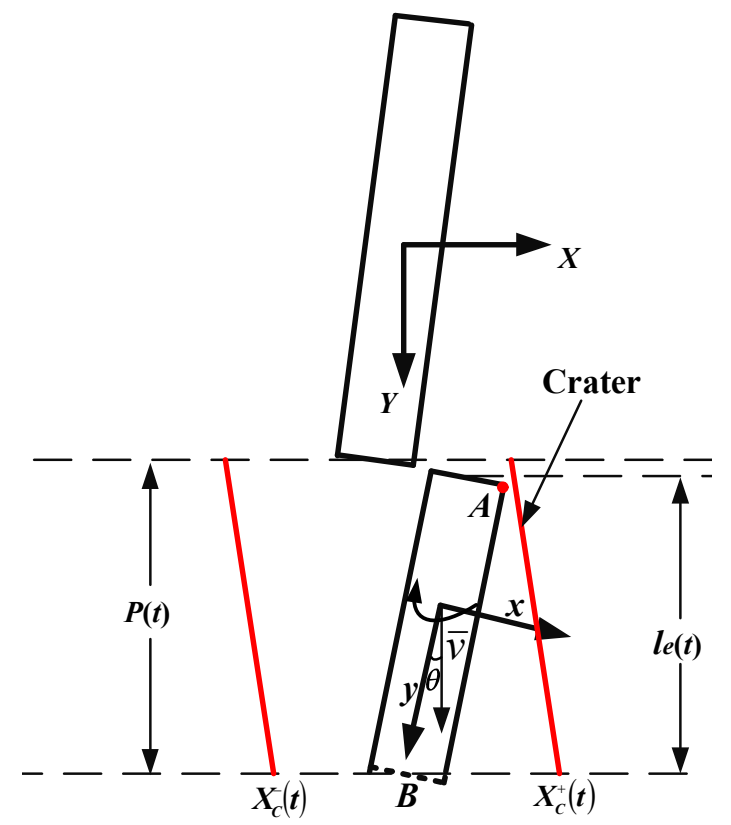

Fig. 1 The orientation and position of the eroding rod.

In general, the solution of Eqs. (1-5) can be obtained numerically. The discrete expressions of Eqs. (1-5) for each time step are given in Appendix 1.

\subsection{Crater shape}

The orientation and position of the eroding rod at time 0 and $t$ are shown in Fig. 1 where $[X, Y]$ and $[x, y]$ represent the fixed global coordinate and the coordinate in a local reference frame located at its current centroid at time $t$, respectively. The transformation from local coordinate to global Paper No. : JAM-16-1374. Corresponding authors: Li and Fang. Page 6 
coordinate is,

$$
\left[\begin{array}{l}
X \\
Y
\end{array}\right]=\left[\begin{array}{cc}
\cos \theta & -\sin \theta \\
\sin \theta & \cos \theta
\end{array}\right]\left[\begin{array}{l}
x \\
y
\end{array}\right]+\left[\begin{array}{l}
X_{0} \\
Y_{0}
\end{array}\right]
$$

where $\quad X_{0}=-\sum_{0}^{t}\left[d l_{e}(t) \tan \theta(t) / 2\right] \quad$ and $\quad Y_{0}=P(t)+L \cos \theta_{0} / 2-l_{e}(t) / 2 \quad$ are the global coordinates of the centroid of the eroding rod at time $t$ where $L$ and $\theta_{0}$ are the original rod length and initial yaw, respectively.

The crater diameter can be determined by different methods [15]. Both energy conservation and momentum conservation methods gave very close predictions for the crater diameter. Based on the momentum conservation during penetration, the crater diameter $D_{c}(t)$ can be determined by [15],

$$
D_{c}(t)=\frac{d}{\cos \theta(t)} \sqrt{\frac{Y_{p}}{R_{t}}+\frac{2 \rho_{p}[v(t)-u(t)]^{2}}{R_{t}}}
$$

As shown in Fig.1, the instantaneous position of the crater sidewall front at time $t$ is $\left(X_{C}^{-}(t), P(t)+L \cos \theta_{0} / 2\right)$ and $\left(X_{C}^{+}(t), P(t)+L \cos \theta_{0} / 2\right)$. It is assumed that the midpoint of the eroding surface of the rod (i.e. point $B$ in Fig.1) is located in the middle of the current crater front. Similar assumption was made in [8] and the calculated crater shape agreed with the corresponding experimental data. With this assumption, one can obtain,

$$
X_{C}^{-}(t)=X_{B}(t)-D_{c}(t) / 2 \quad X_{C}^{+}(t)=X_{B}(t)+D_{c}(t) / 2
$$

From Fig. 1 and Eq. (6), $X_{B}(t)$ is given by,

$$
X_{B}(t)=-\tan \theta(t) l_{e}(t) / 2-\sum_{0}^{t}\left[d l_{e}(t) \tan \theta(t) / 2\right]
$$

Then the crater outline can be determined according to Eqs. $(7,8)$ at the end of each time step. 


\subsection{Determination of the critical yaws}

Relative positions of the rod and crater sidewalls are shown in Fig.2. Two critical yaw angles $\left(\theta_{c 1}\right.$ and $\left.\theta_{c 2}\right)$ are defined in the present paper. When the initial yaw angle is less than $\theta_{c 1}$, no contact between the rod and crater sidewall (including the crater entrance) occurs during the entire penetration. Otherwise, if the initial yaw angle is greater than $\theta_{c 1}$, the rod end will collide with some points of the crater sidewall during penetration. The second critical yaw angle $\theta_{c 2}$ is defined in the same way as $\theta_{c 1}$ for the collision of rod body with the crater entrance during penetration. It is evident that $\theta_{c 1} \leq \theta_{c 2}$ according to their definitions. $\theta_{c 2}$ is more important than $\theta_{c 1}$ because, if striking yaw angle $\left(\theta_{0}\right)$ is greater than $\theta_{c 2}$, collision of the rod with the crater entrance will produce a large bending moment to break the rod, and as a consequence, DOP will decrease significantly. This section focuses on the determination of the $\theta_{c 1}$ and $\theta_{c 2}$ with further discussion about these two critical yaws given in Sec. 4.1.

The positions of rod and crater sidewalls can be determined based on the procedure described in Sec. 2.2. Generally, the critical yaws can be found by inputting a series of initial yaws and comparing the relative positions of the rod and crater sidewalls until the critical conditions are satisfied. However, this method is time-consuming and not accurate. Thus, a contact function and iterative algorithm are used for the determination of critical yaws in this section. 


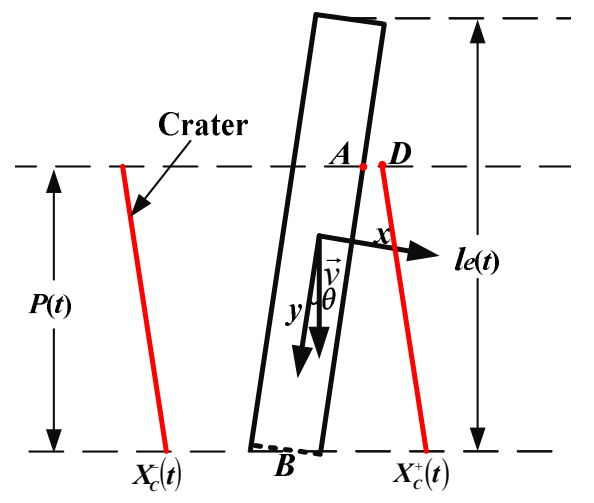

(a) $P(t)<l_{e}(t)$

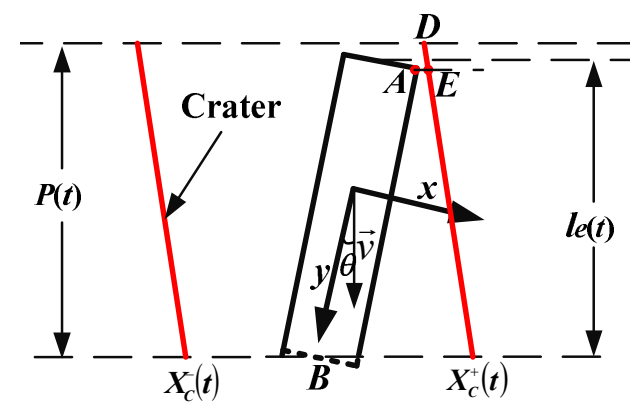

(b) $P(t) \geq l_{e}(t)$

Fig.2 Locations of the rod and crater sidewalls.

If the instantaneous DOP is less than instantaneous $l_{e}(t)$, the contact function for $\theta_{c 1}$ is defined as

$$
f_{1}=\max \left[X_{A}(t)-X_{D}\right]
$$

where points $A$ and $D$ have the same Y-coordinates (i.e. they are on the same level), and $X_{A}(t)$ and $X_{D}$ are the global X-coordinates of points $A$ and $D$, as shown in Fig.2. Point $D$ denotes the crater entrance and point $A$ is the closest point of the rod to point $D$ when they are on the same level.

When the instantaneous DOP is greater than instantaneous $l_{e}(t)$, the contact function for $\theta_{c 1}$ is defined as follows

$$
f_{1}=\max \left[X_{A}(t)-X_{E}(t)\right]
$$

where point $A$ is now the point at the top-right edge of the rod and point $E$ is the point on the crater sideway and on the same level of point $A$, as shown in Fig.2. Using the coordinate transformation formula (Eq. (6)), $X_{A}(t), X_{D}$ and $X_{E}(t)$ can be determined, i.e.

$$
X_{A}(t)= \begin{cases}d \cos \theta(t) / 2-\left[l_{e}(t) / 2-P(t)\right] \tan \theta(t)-\sum_{0}^{t}\left[d l_{e}(t) \tan \theta(t) / 2\right], & P(t) \leq l_{e}(t) \\ d \cos \theta(t) / 2+\tan \theta(t) l_{e}(t) / 2-\sum_{0}^{t}\left[d l_{e}(t) \tan \theta(t) / 2\right], & P(t)>l_{e}(t)\end{cases}
$$




$$
X_{D}=X_{C}^{+}(0), X_{E}(t)=X_{c}^{+}\left(t_{1}\right)
$$

where $t_{1}$ is implicitly related to $t$ by the point $\left(X_{C}^{+}\left(t_{1}\right), Y_{C}^{+}\left(t_{1}\right)\right)$ on the crater sideway when $Y_{C}^{+}\left(t_{1}\right)=L \cos \left(\theta_{0}\right) / 2+P(t)-l_{e}(t)+d \sin \theta(t) / 2$ (note: $t_{1}$ represents the time when the front of the crater sideway reaches this point). Then the contact function for $\theta_{c 1}$ can be expressed explicitly as,

$$
f_{1}=\max \begin{cases}d \cos \theta(t) / 2-\left[l_{e}(t) / 2-P(t)\right] \tan \theta(t)-\sum_{0}^{t}\left[d l_{e}(t) \tan \theta(t) / 2\right]-X_{C}^{+}(0), & P(t) \leq l_{e}(t) \\ d \cos \theta(t) / 2+\tan \theta(t) l_{e}(t) / 2-\sum_{0}^{t}\left[d l_{e}(t) \tan \theta(t) / 2\right]-X_{c}^{+}\left(t_{1}\right), & P(t)>l_{e}(t)\end{cases}
$$

It is evident that $f_{1}=0$ when the initial yaw is equal to $\theta_{c 1}$. So an iterative algorithm for the determination of this critical yaw is proposed as follows,

$$
\theta_{0}(i+2)=\frac{-f_{1}(i)}{f_{1}(i+1)-f_{1}(i)}\left[\theta_{0}(i+1)-\theta_{0}(i)\right]+\theta_{0}(i)
$$

Given two initial values for $\theta_{0}$ and $f_{1}$, the critical yaw $\theta_{c 1}$ can be obtained from Eq. (15) until $\left|f_{1}\right|$ is less than a prescribed threshold (e.g. 1e-10). Usually, the critical yaw can be found in less than five iterative steps.

Similarly, the contact function for $\theta_{c 2}$ is

$$
f_{2}=\max \left\{d \cos \theta(t) / 2-\left[l_{e}(t) / 2-P(t)\right] \tan \theta(t)-\sum_{0}^{t}\left[d l_{e}(t) \tan \theta(t) / 2\right]-X_{C}^{+}(0)\right\}, P(t) \leq l_{e}(t)
$$

The method to determine $f_{2}$ is the same as that used to determine $f_{1}$, which will not be repeated here.

\section{Verification of the proposed model}

In this section, results from the present model are compared with corresponding experiments Paper No. : JAM-16-1374. Corresponding authors: Li and Fang. Page 10 
and empirical formula. Penetration rods and targets are made from tungsten alloys and RHA, respectively. Material properties are $\rho_{p}=17300 \mathrm{~kg} / \mathrm{m}^{3}, \rho_{t}=7850 \mathrm{~kg} / \mathrm{m}^{3}, \quad Y_{p}=2.3 G P a$ and $R_{t}=6.5 G P a[16]$.

\subsection{Crater shape}

The predicted crater shape and locations of the rod at different time are shown in Fig. 3(a), where the striking velocity is $1600 \mathrm{~m} / \mathrm{s}$, initial yaw is $2.5^{\circ}$ and length-diameter ratio is 10 . Fig. 3(b) presents the schematic crater shape according to experimental observation [3]. It can be found that the predicted crater shape is similar to a mirror reflected image of the long rod about the target surface line, which agrees with the experimental observations shown in Fig. 3(b) [3].

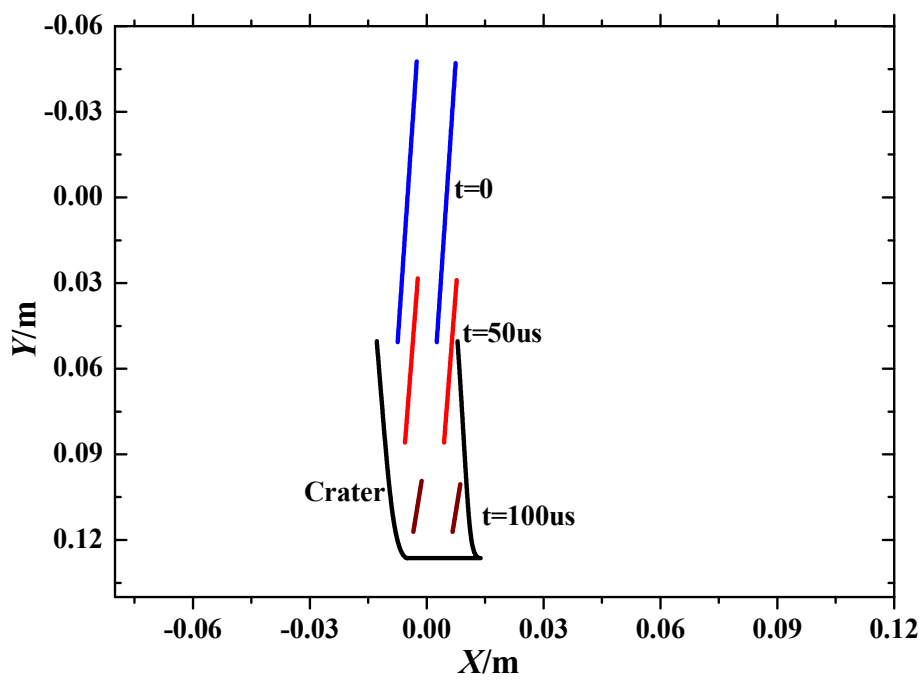

(a) Predicted crater shape

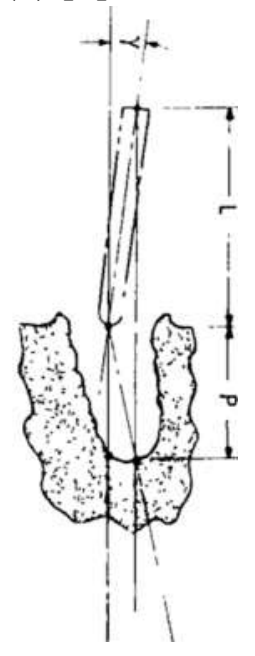

(b) Experimental observation [3]

Fig.3 Comparison of the calculated crater shape with experimental observation from [3].

\subsection{Comparison with experimental data}

Fig. 4(a) presents the experimental results for $V=1400 \mathrm{~m} / \mathrm{s}$ and $L / d=10$ [17] together with the predicated critical yaw angle and simulation results obtained by Rosenberg et al. [9]. Although there are limited experimental data for small yaw angles, the predicted critical yaw $\left(2.6^{\circ}\right)$ agrees well with Paper No. : JAM-16-1374. Corresponding authors: Li and Fang. Page 11 
both experimental and simulation results. Fig.4(b) gives the comparison of the predicted critical yaw angle $\left(1.5^{\circ}\right)$ with experimental results for $V=1650 \mathrm{~m} / \mathrm{s}, L / d=20$ [18]. Reasonably good agreement is observed although the predicted critical yaw seems smaller than the actual one, which may be attributed to the uncertainty of experimental measurement of the small yaw and the inaccuracy of material parameters of penetration rod and target $\left(Y_{p}\right.$ and $\left.R_{t}\right)$.

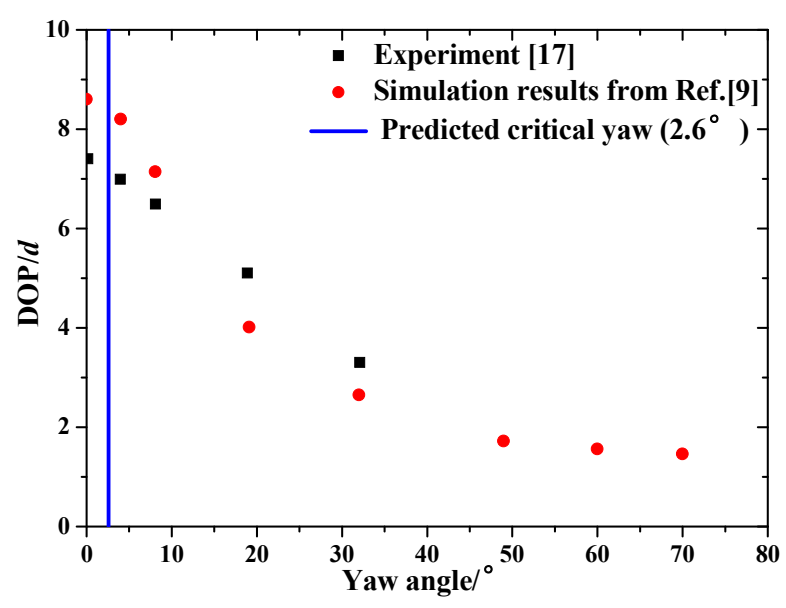

(a) $V=1400 \mathrm{~m} / \mathrm{s}, L / d=10[9,17]$

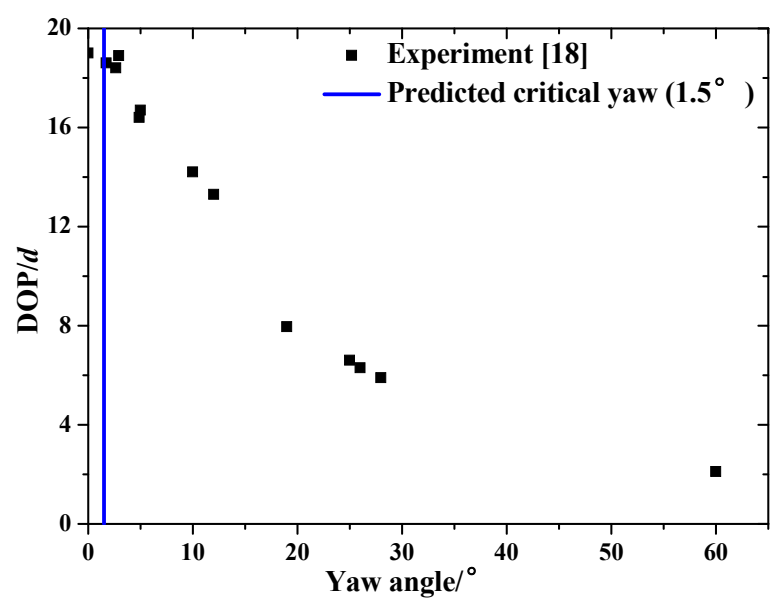

(b) $V=1650 \mathrm{~m} / \mathrm{s}, L / d=20[18]$

Fig.4 Comparison of the predicted yaw angle with experiments and simulation results.

\subsection{Comparison with the empirical formula}

Bjerke et al. [3] proposed an empirical formula of the critical yaw for penetration of RHA targets and tungsten-alloy penetrators.

$$
\begin{aligned}
\theta_{c} & =\sin ^{-1}\left[\left(D_{c}-d\right) / 2 L\right] \\
D_{c} / d & =1.1524+0.3388 V+0.1286 V^{2}
\end{aligned}
$$

where the unit of the impact velocity $V$ is $\mathrm{km} / \mathrm{s}$ and the critical yaw $\theta_{c}$ is measured by radian, respectively. Comparisons of the predicted initial yaw angle with this empirical formula for different velocities with $L / d$ ratios of 10, 20, 30 are shown in Fig.5. The calculated results when the rod rotation is not considered (i.e. yaw angle is a constant during penetration) are also presented in Fig.5. Paper No. : JAM-16-1374. Corresponding authors: Li and Fang. Page 12 
It is found that the predicted yaw angle agrees well with the empirical formula in the low velocity range, while discrepancy exists when striking velocity is high. The calculated critical yaws when the rod rotation is neglected are always bigger than those when the rod rotation is considered. The difference between these two critical yaws due to the consideration of rod rotation decreases with the increase of the striking velocity.

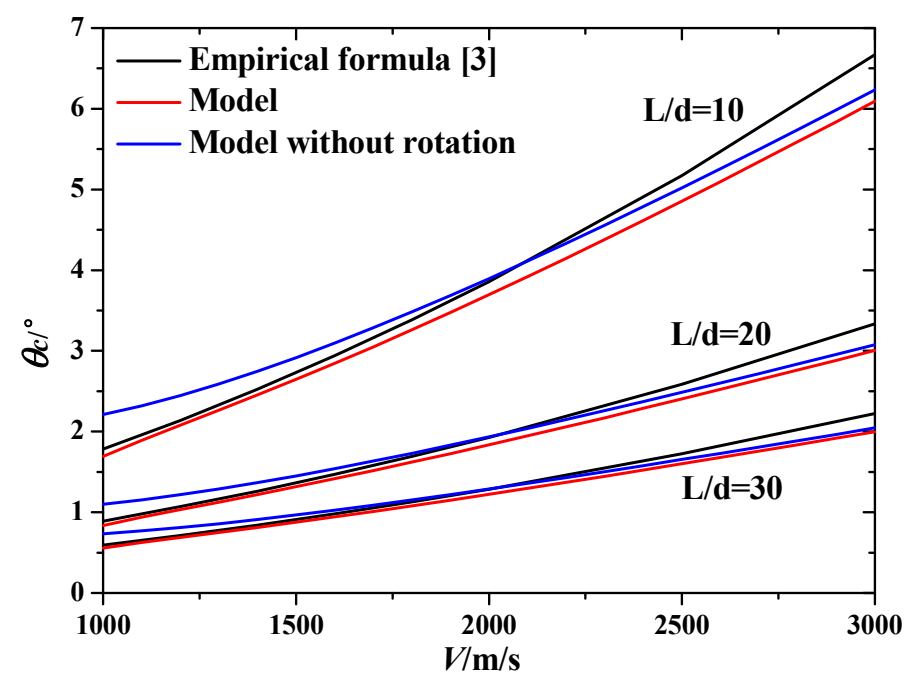

Fig.5 Comparison of the predicted critical yaw angle with empirical formula [3].

Variation of instantaneous yaw angle during the penetration is also of interest. Yaw angle versus time for different $L / d$ ratios and velocities are shown in Fig. 6. It can be observed that the change of yaw angle increases with the increase of velocity and with the decrease of $L / d$. It is evident that the instantaneous effective rod length $l_{e}$ decreases with the increase of striking velocity and with the decrease of $L / d$. Thus, the angular acceleration and change of yaw angle increases with the increase of velocity and the decrease of $L / d$ according to Eq. (5). 


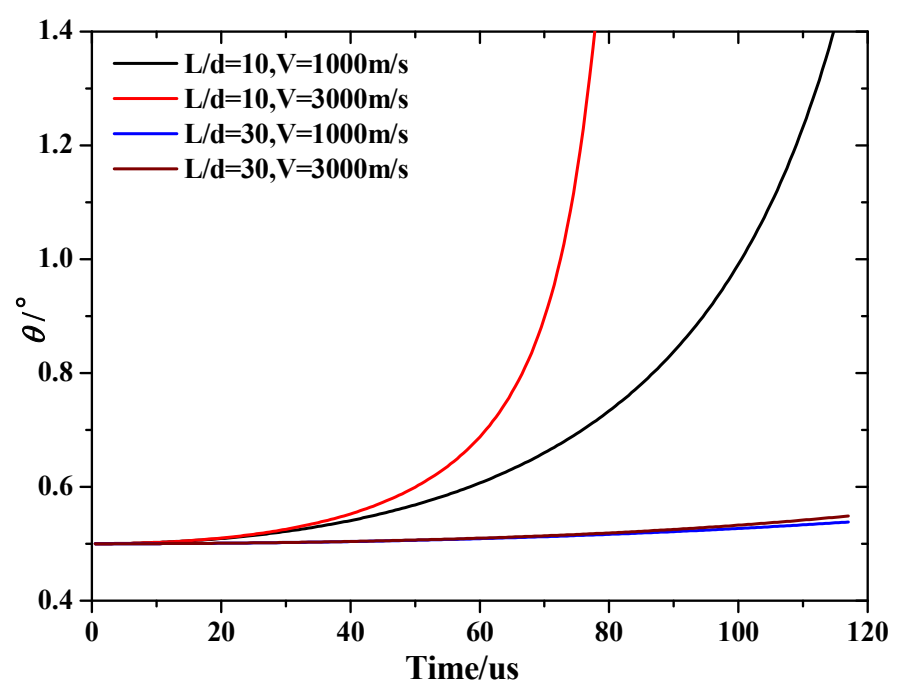

Fig. 6 Variation of yaw angel during the penetration.

\section{Studies of three typical cases}

There are four non-dimensional numbers that govern the critical yaws, i.e. $\lambda=R_{t} / Y_{p}$, $\varsigma=\frac{\rho_{p} V^{2}}{Y_{p}}, \mu=\sqrt{\frac{\rho_{t}}{\rho_{p}}}$ and $\psi=d / L$. For given rod and target materials, $\mu$ is a constant. This section will focus on the effects of $\lambda, \varsigma$ and $\psi$ on the two critical yaws $\theta_{c 1}$ and $\theta_{c 2}$.

\subsection{Tungsten alloy rod penetrating steel targets}

The material properties of tungsten alloy rod are considered as constants $\left(\rho_{p}=17300 \mathrm{~kg} / \mathrm{m}^{3}\right.$, $\left.Y_{p}=2.32 \mathrm{GPa}[16]\right)$ while the hardness and dynamic yield strength of steel targets are changed. Referring to the penetration data in Ref. [19] and considering the actual situation, the range of the BHN hardness of steel targets is 200-400 (including C110W1, C110W2, St52, 4340, armor steels, etc. [19]). Using the formula for $Y_{p}$ and $R_{t}$ proposed by Tate [16],

$$
\begin{gathered}
Y_{p}=1.7 \sigma_{y p} \quad \sigma_{y p}=4.2 \times B H N(M P a) \\
R_{t}=\sigma_{y t}\left[2 / 3+\ln \left(0.57 E_{t} / \sigma_{y t}\right)\right] \sigma_{y t}=4.2 \times B H N(M P a)
\end{gathered}
$$

$R_{t}$ is found to be $4.7 \mathrm{GPa}-8.3 \mathrm{Gpa}$ where $\sigma_{y p}$ and $\sigma_{y t}$ are the dynamic yield strengths of the projectile and target, respectively. $E_{t}$ is the elastic modulus of the target. Thus, $\lambda$ is ranged from 2 Paper No. : JAM-16-1374. Corresponding authors: Li and Fang. Page 14 
to 3.6. The calculated range of Johnson's damage number $\varsigma$ is $8-67$ for the striking velocities from $1000 \mathrm{~m} / \mathrm{s}$ to $3000 \mathrm{~m} / \mathrm{s} . \psi=d / L$ are $1 / 10,1 / 20$ and $1 / 30$, respectively. The influences of $\lambda, \varsigma$ and $\psi$ on two critical yaws $\left(\theta_{c 1}\right.$ and $\left.\theta_{c 2}\right)$ are shown in Fig.7. It can be observed that the critical yaws increase with the decrease of $\lambda$ and with the increases of $\varsigma$ and $\psi . \theta_{c 1}$ is smaller than $\theta_{c 2}$ for low values of $\lambda$ and $\varsigma$ while they are equal as $\lambda$ and $\varsigma$ become large values.

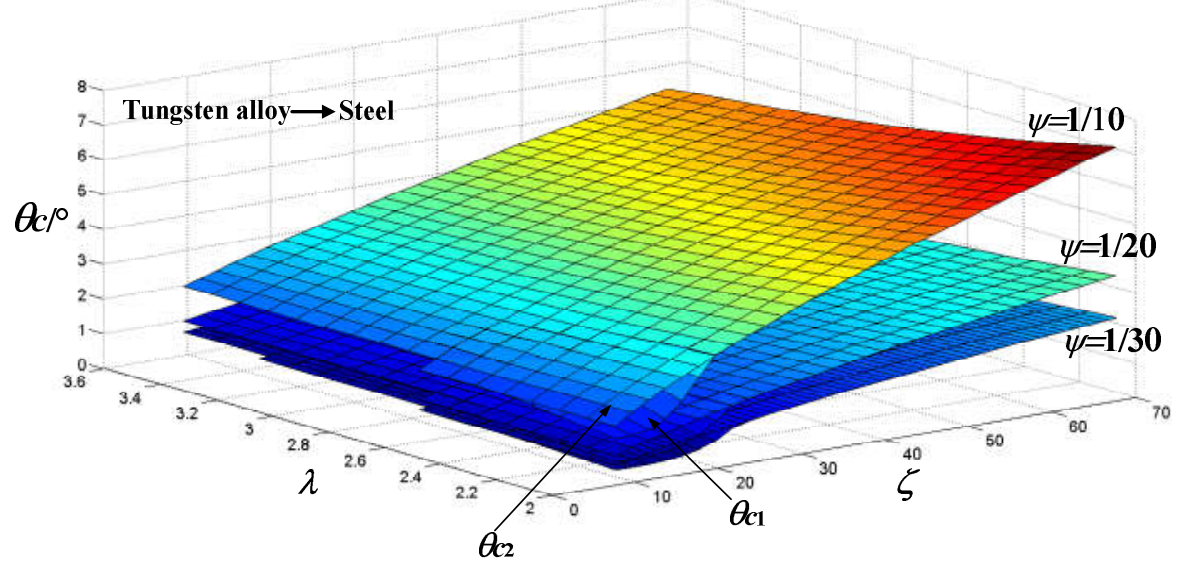

Fig.7 The influence of $\lambda, \varsigma$ and $\psi$ on critical yaws for a given tungsten alloy rod and steel targets.

In order to gain some insight into the relationship between $\theta_{c 1}$ and $\theta_{c 2}$, a calculated example is shown in Fig. 8, in which $\lambda=2, \psi=1 / 10$ and striking velocities are in the range from $1000 \mathrm{~m} / \mathrm{s}$ to $3000 \mathrm{~m} / \mathrm{s}$. It can be found that $\theta_{c 1}$ is smaller than $\theta_{c 2}$ if the striking velocity is below $1600 \mathrm{~m} / \mathrm{s}$. In this region, if the striking yaw angle is smaller than $\theta_{c 1}$, the contact of rod with crater sidewall will not happen. If the striking yaw angle is between $\theta_{c 1}$ and $\theta_{c 2}$, the rod will not hit the crater entrance, but will collide with the crater sidewall. Under this circumstance, DOP may not be decreased markedly since the rod tail only marginally interferes with the crater sidewall. The rod tail will ricochet gently from the sidewall without breaking [3]. However, if the striking yaw angle is greater than $\theta_{c 2}$, then the rod body will collide with the crater entrance, producing a great bending Paper No. : JAM-16-1374. Corresponding authors: Li and Fang. Page 15 
moment which may break the rod, and consequently DOP will decrease significantly.

Another interesting phenomenon shown in Fig. 8 is that $\theta_{c 1}$ is equal to $\theta_{c 2}$ when the striking velocity is beyond $1600 \mathrm{~m} / \mathrm{s}$. This means that, at least for the tungsten alloy rod and steel targets presented here, if rod does not collide with the crater entrance, then the interference of the rod with crater sidewall will not happen during the penetration when the striking velocity is larger than $1600 \mathrm{~m} / \mathrm{s}$.

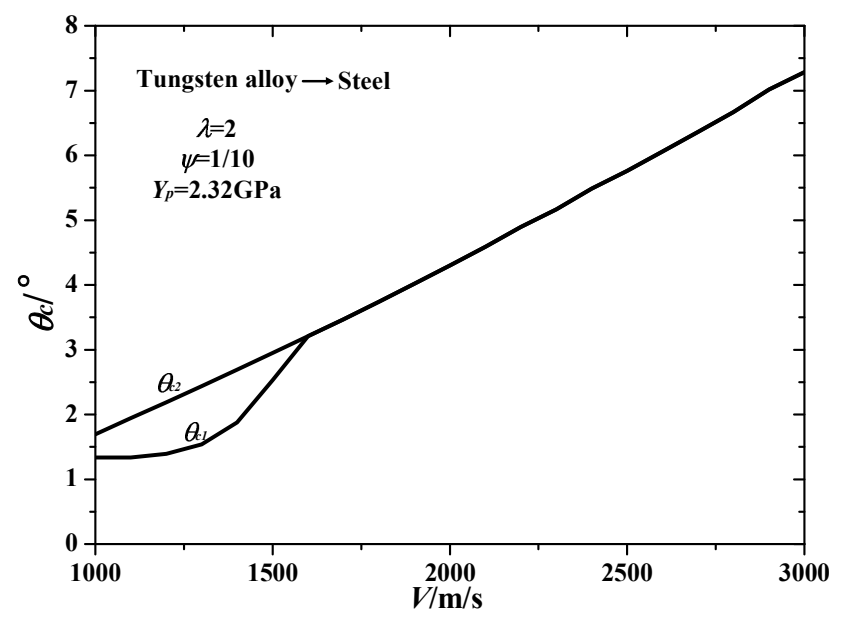

Fig.8 Relationship between $\theta_{c 1}$ and $\theta_{c 2}$ for $\lambda=2, \psi=1 / 10$ at different striking velocities.

\subsection{Steel rods penetrating aluminum alloy target}

In this section, we consider steel rods penetrating a given aluminum alloy target (7075-T6 Al, $\rho_{p}=2700 \mathrm{~kg} / \mathrm{m}^{3}, \mathrm{BHN}=145$ [19] (page A-41)). The range of the BHN hardness of steel rods is 110-295 (including C1015, St37, St52, 4340 steels, etc. [19]). Using Eq. (18), $R_{t}$ and $Y_{p}$ are found to be $2.9 \mathrm{GPa}$ and $0.8 \mathrm{GPa}-2 \mathrm{GPa}$. The striking velocities are between $1000 \mathrm{~m} / \mathrm{s}$ and $3000 \mathrm{~m} / \mathrm{s}$. Thus, the ranges of $\lambda$ and $\varsigma$ are 1.45-3.6 and 4-88, respectively. $d / L$ ratios considered are also $1 / 10,1 / 20$ and $1 / 30$.

The influence of $\lambda, \varsigma$ and $\psi$ on the critical yaws is shown in Fig. 9. Similar trends to those 
in Fig. 7 are observed. It is interesting to note that $\theta_{c 1}$ is very small when $\lambda$ approaches to unity, which means that, in this case, it is difficult to avoid the interference of rod with crater sidewall during penetration.

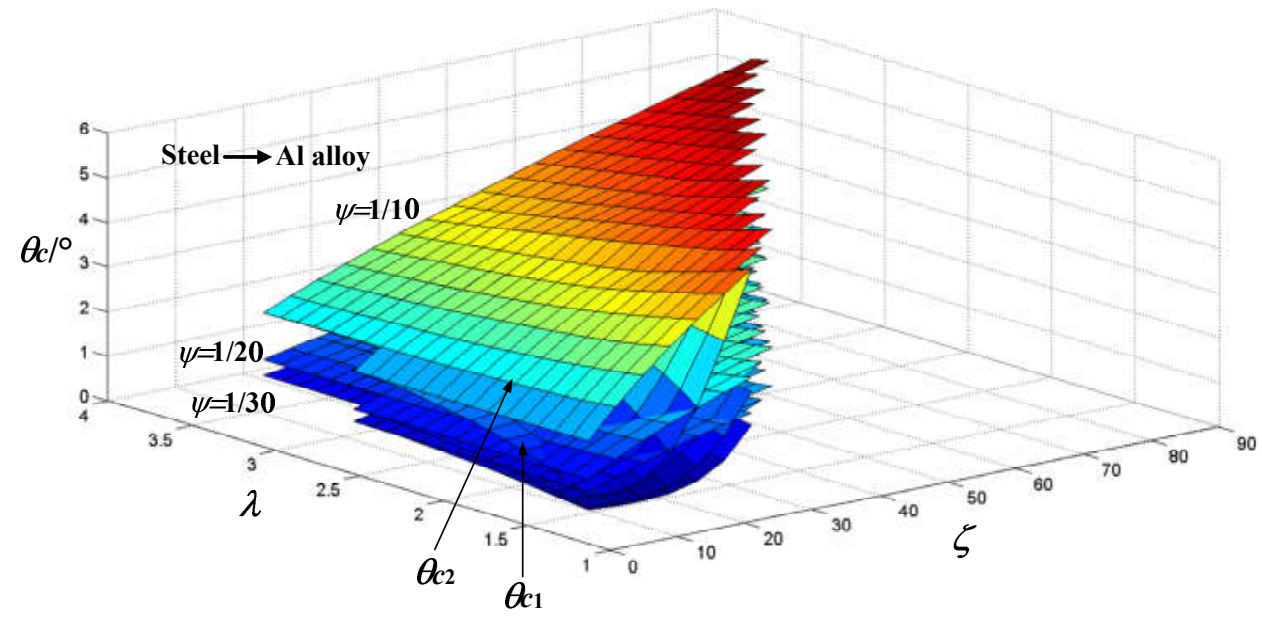

Fig.9 The influence of $\lambda, \varsigma$ and $\psi$ on critical yaws for steel rods and a given aluminum alloy target.

\subsection{Steel rods penetrating steel targets}

This is the case that both the material properties of rod and target may vary. The BHN hardness range of steel rods and targets is still 200-400, including C110W1, C110W2, St52, 4340, armor steels, etc. [19]. Using Eq. (18), the range of $R_{t}$ and $Y_{p}$ are found to be $4.7 \mathrm{GPa}-8.3 \mathrm{GPa}$ and $1.4 \mathrm{GPa}-2.8 \mathrm{GPa}$, respectively. The striking velocities are still between $1000 \mathrm{~m} / \mathrm{s}-3000 \mathrm{~m} / \mathrm{s}$. Therefore, the range of $\lambda$ and $\varsigma$ considered are about 1.6-6 and 5-50, respectively. $d / L$ ratios considered are also $1 / 10,1 / 20$ and $1 / 30$.

Fig. 10 presents the influence of $\lambda, \varsigma$ and $\psi$ on critical yaws. Similar trend to those in Figs. 7 and 9 are observed. No further discussion is given. 


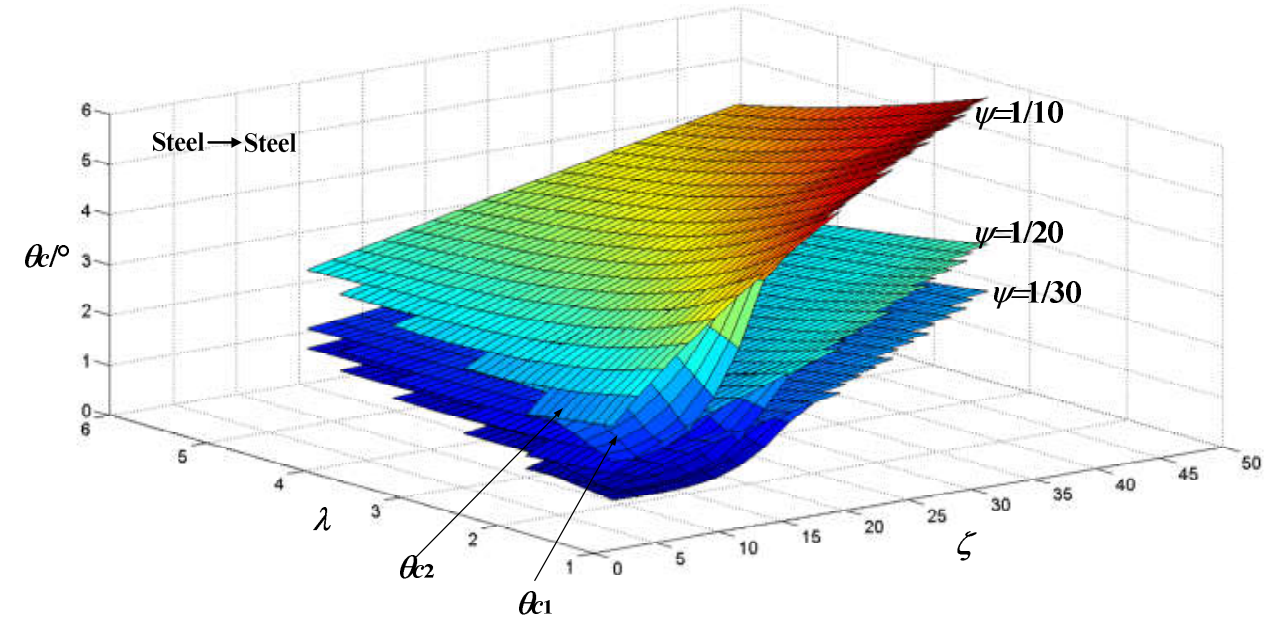

Fig.10 The influence of $\lambda, \varsigma$ and $\psi$ on critical yaws for steel rods and steel targets.

\section{New empirical formula for critical yaw}

Based on previous three typical cases, we propose an empirical formula for the critical yaw $\theta_{c 2}$ based on the four non-dimensional numbers recommended in Sec. 4, i.e.

$$
\theta_{c 2}=\frac{\psi}{2+3 / \varsigma} \sqrt{\frac{1}{\lambda}+2 \frac{\varsigma}{\lambda}\left[\frac{\mu^{2}-\sqrt{\mu^{2}+2(\lambda-1)\left(1-\mu^{2}\right) / \varsigma}}{1-\mu^{2}}\right]^{2}}-\frac{\psi}{2+3 / \varsigma}
$$

When $\mu$ approaches unity, the denominator $\left(1-\mu^{2}\right)$ tends to zero. Equation (19) can be simplified to,

$$
\theta_{c 2}=\frac{\psi}{(2+3 / \varsigma)} \sqrt{\frac{1}{\lambda}+\frac{2 \varsigma}{\lambda}\left(\frac{1-\lambda}{\varsigma}-\frac{1}{2}\right)^{2}}-\frac{\psi}{(2+3 / \varsigma)}
$$

The empirical condition for $\theta_{c 1}=\theta_{c 2}$ is proposed as

$$
\varsigma \lambda^{3} \psi^{0.2} \geq 100
$$

Comparisons of Eq. (19) (or Eq. (20)) with the three typical cases in Sec. 4 are shown in Fig. 11, in which the green surfaces represent the critical yaws predicted by Eq. (19) (or Eq. (20)), while the red surfaces denote the results from the analytical model. Fig. 12 also shows the comparison between Eq. (19) (or Eq. (20)) with the empirical formula proposed in Ref. [3] with excellent agreement. The Paper No. : JAM-16-1374. Corresponding authors: Li and Fang. Page 18 
present empirical formulae (Eqs. $(19,20))$, which are expressed using non-dimensional physical numbers, have wider application range than the empirical formula proposed in Ref. [3].

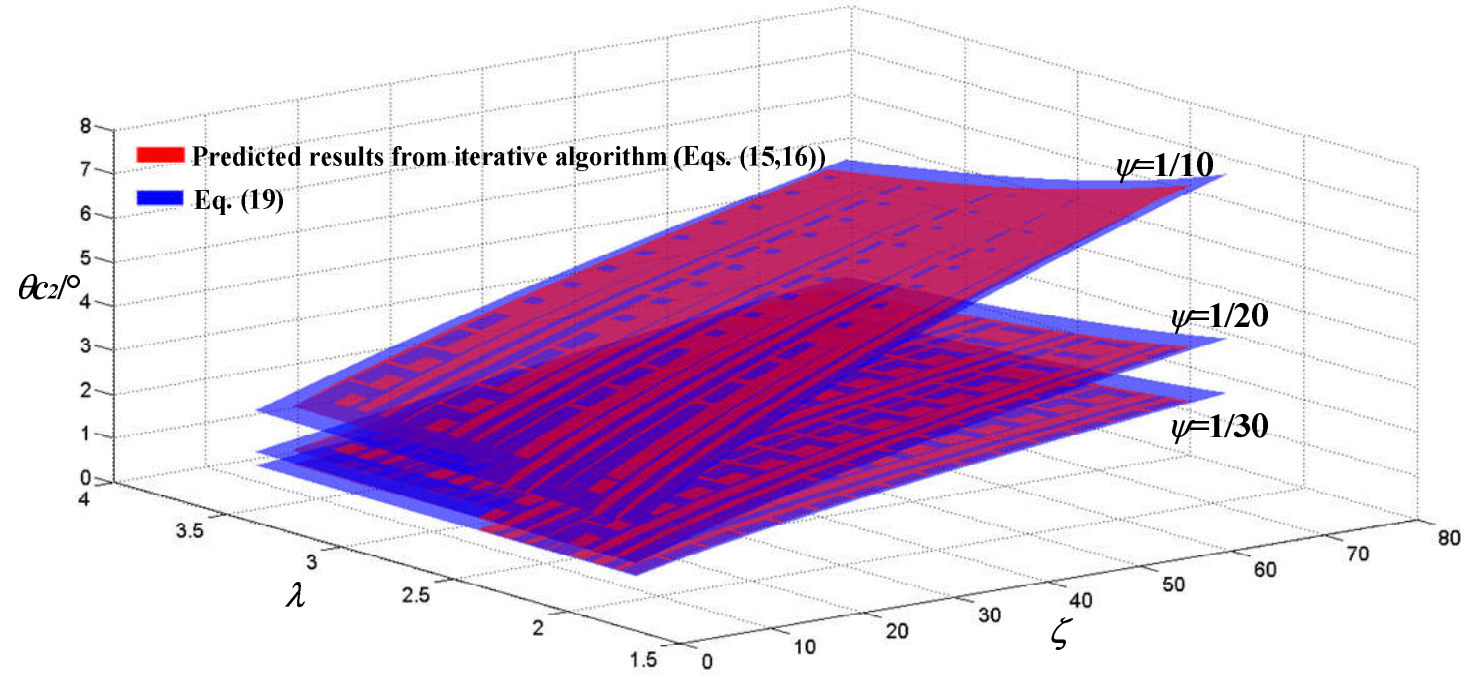

(a) Tungsten alloy rod penetrating steel targets

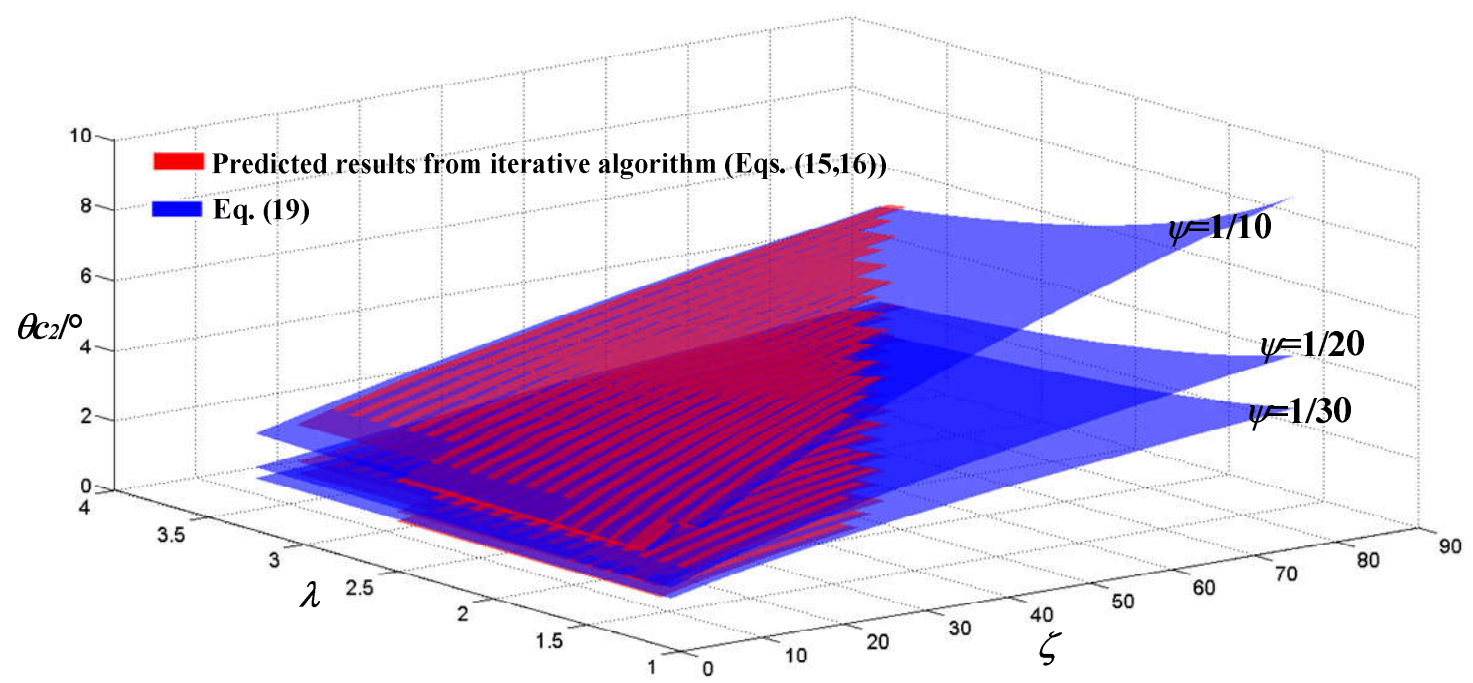

(b) Steel rods penetrating aluminum alloy target 


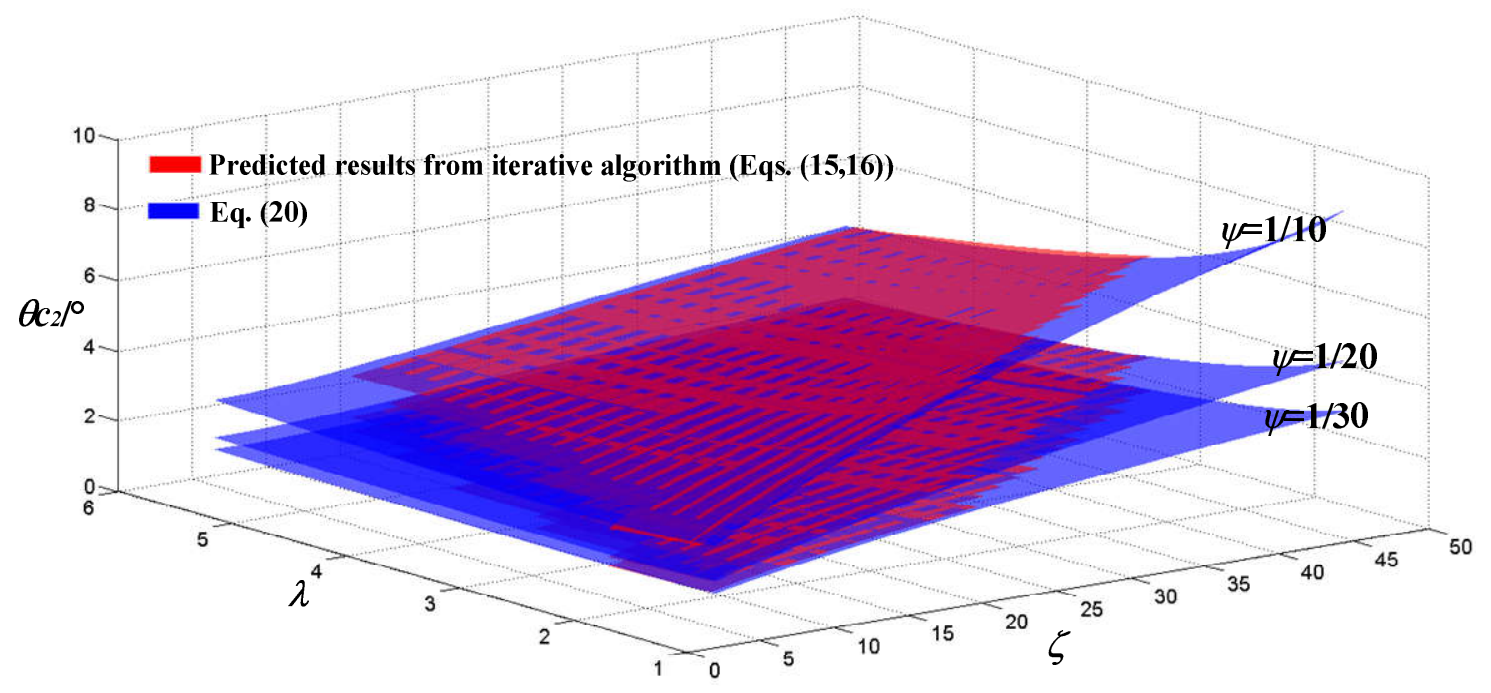

(c) Steel rods penetrating steel targets

Fig.11 Comparisons of the new empirical formula with the predicted results from iterative algorithm

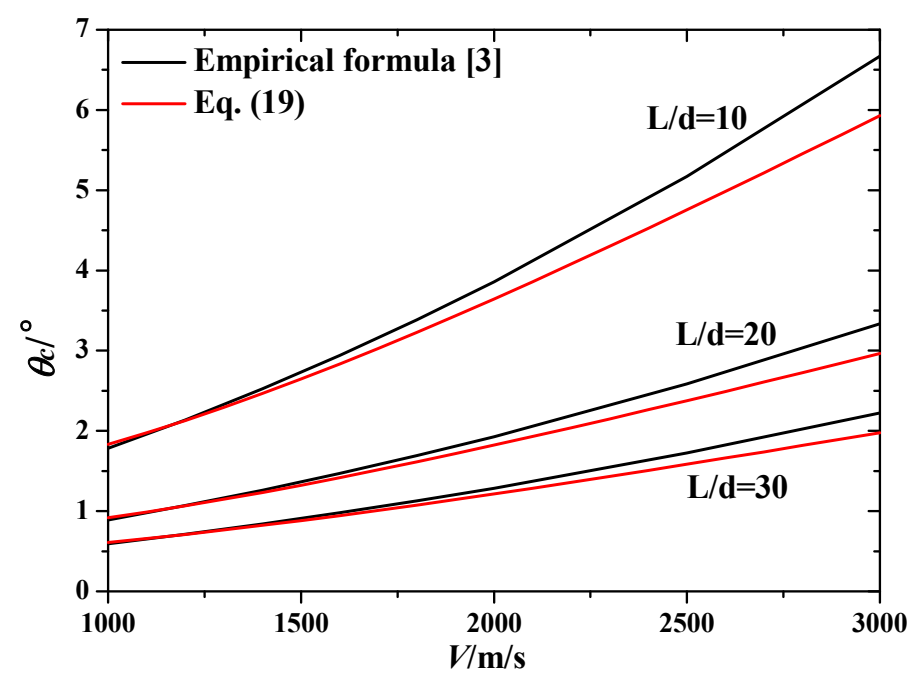

Fig. 12 Comparisons of the new empirical formula Eq. (19) with the empirical formula proposed in Ref. [3].

\section{Conclusions}

An improved model for yawed normal penetration of a long rod is presented. The model considers the deceleration and rotation of the rod and is capable of predicting the penetration crater when the rod body has no contact with the crater during the penetration. Using the proposed model Paper No. : JAM-16-1374. Corresponding authors: Li and Fang. Page 20 
and contact conditions, two critical yaws were identified, below which contacts between the rod and crater sidewall and between the rod and the crater entrance during the entire penetration do not occur. The second critical yaw is important for the design of long rod penetration.

It was also found that four non-dimensional numbers (i.e. the ratio of the target resistance to the rod strength $\lambda$, Johnson's damage number of the $\operatorname{rod} \varsigma$, the diameter-length ratio of the rod $\psi$, and the square root of the density ratio $\mu$ ) are dominant for the determination of the critical yaws. The critical yaws increases with the decrease of $\lambda$ and the increases of $\varsigma$ and $\psi$. It was found that two critical yaw angles coincide when $\varsigma \lambda^{3} \psi^{0.2} \geq 100$. A new empirical formula based on these non-dimensional numbers and parametric analyses is proposed, which agrees well with existing experimental, numerical and empirical results and has wider application range.

\section{Acknowledgements}

This study was supported by the National Natural Science Foundations of China for Creative Research Groups of China (51321064, 11221202), and the National Natural Science Foundations of China $(51378015,11390362)$.

\section{References}

[1] Tate, A., 1967, “A theory for the deceleration of long rods after impact,” J. Mech. Phys. Solids 15(6), pp. 387-399.

[2] Alekseevskii, V.P., 1966, "Penetration of a rod into a target at high velocity," Combust. Explos. Shock Waves 2, pp. 63-66. 
[3] Bjerke, T.W., Silsby, G.F., Scheffler, D.R., and Mudd, R.M., 1992, "Yawed long-rod armor penetration,” Int. J. Impact Engng 12(2), pp. 281-292.

[4] Bless, S.J., Barber, J.P., Bertke, R.S., and Swift, H.F., 1978, "Penetration mechanics of yawed rods,” Int. J. Engng Sci. 16, pp. 829-834.

[5] Walker, J.D., Anderson, Jr. C.E., and Goodlin, D.L., 2001, "Tungsten into steel penetration including velocity, L/D, and impact inclination effects," $19^{\text {th }}$ Int. Symp. Ballistics, Interlaken, Switzerland, pp. 1133-1139.

[6] Yaziv, D., Walker, J.D., and Riegel, J.P., 1992, “Analytical model of yawed penetration in the 0 to 90 degrees range," Proc. $13^{\text {th }}$ Int. Symp. Ballistics, Stockholm, Sweden, pp. 17-23.

[7] Bukharev, Y. I., and Zhukov, V.I., 1995, "Model of the penetration of a metal barrier by a rod projectile with an angle of attack," Combust. Explos. Shock Waves 31(3), pp. 362-367.

[8] Lee, M., 2000, “An engineering impact model for yawed projectiles,” Int. J. Impact Engng, 24(8), pp. 797-807.

[9] Rosenberg. Z., Dekel, E., and Ashuach, Y., 2006, "More on the penetration of yawed rods," J. Phys. IV France 134, pp. 397-402.

[10] Bless, S.J., Satapathy, S., and Normandia, M.J., 1999, “Transverse loads on a yawed projectile,” Int. J. Impact Engng 23(1), pp. 77-86.

[11] Bless, S.J., Tarcza, K., Taleff, E., Russell, R., and Huerta, M., 2005, “Deflection and fracture of tungsten rods by yawed impact," Proc. $22^{\text {th }}$ Int. Symp. Ballistics, San Antonio, BC, pp. 1163-1170.

[12] Tate, A., 1969, "Further results in the theory of long rod penetration,” J. Mech. Phys. Solids 17, pp. 141-150. 
[13] Rosenberg, Z., and Dekel, E., 2012, “Terminal ballistics,” Berlin: Springer.

[14] Anderson, Jr. C.E., Walker, J.D., and Hauver, G.E., 1992, “Target resistance for long-rod penetration into semi-infinite targets,” Nuclear Eng. Des. 138(1), pp. 93-104.

[15] “Cavity dynamics for long rod penetration," IAT Report 0094, Institute for Advance Technology, Austin, 1996.

[16] Tate, A., 1986, "Long rod penetration models-Part II: Extensions to the hydrodynamic theory of penetration,” Int. J. Engng Sci. 28(9), pp. 599-612.

[17] Yaziv, D., Rosenberg, Z., and Riegel, J.P., 1990, "Penetration capability of yawed long rod penetrators," Proc. $12^{\text {th }}$ Int. Symp. Ballistics, San Antonio, pp. 202-207.

[18] Hohler, V., and Behner, T., 1999, "Influences of the yaw angle on the performance reduction of long rod projectiles,” Proc. 18th Int. Symp. Ballistics, San Antonio, TX, pp. 931-938.

[19] Anderson, Jr. C.E., Morris, B.L., and Littlefield, D.L., 1992, “A penetration mechanics database," SwRI Report 3593/001, AD-A246351, Southwest Research Institute, San Antonio, TX.

\section{Appendix 1}

The discrete expressions of Eqs. (1-5) are

$$
\begin{aligned}
& d v(t)=-\frac{Y_{p}}{\rho_{p} l_{e}(t)} d t, \quad v(t+d t)=v(t)+d v(t), \\
& u(t+d t)=\frac{-\rho_{p} v(t+d t)+\sqrt{2\left(\rho_{p}-\rho_{t}\right)\left(R_{t}-Y_{p}\right)+\rho_{p} \rho_{t} v(t+d t)^{2}}}{\rho_{p}-\rho_{t}}, \quad \ddot{\theta}(t)=\frac{Y_{p} \tan \theta(t)}{\rho_{p}\left(\frac{l_{e}(t)^{2}}{6 \cos ^{2} \theta(t)}+\frac{d^{2}}{8}\right)}, \\
& d \dot{\theta}(t)=\ddot{\theta}(t) d t
\end{aligned}
$$


where $d v(t)$ and $d \dot{\theta}(t)$ are the incremental velocity and angular velocity, respectively. $d t$ represents the time step. Variables can be updated at the end of each time step (considering the rotation motion is uniformly accelerated) as follows,

$d l_{e}(t)=[u(t)-v(t)] d t, \quad d P(t)=u(t) d t, \quad \dot{\theta}(t+d t)=\dot{\theta}(t)+d \dot{\theta}(t), \quad d \theta(t)=\dot{\theta}(t) d t+\ddot{\theta}(t) d t^{2} / 2$, $\theta(t+d t)=\theta(t)+d \theta(t), P(t+d t)=P(t)+d P(t), l_{e}(t+d t)=l_{e}(t)+d l_{e}(t), \quad t=t+d t$

where $d l_{e}(t), d P(t)$ and $d \theta(t)$ are the incremental effective length, DOP and yaw angle, respectively. Then penetration event can be numerically solved using Eqs. (A1-A2) with the initial condition $v(0)=V, \theta(0)=\theta_{0}$, and $l_{e}(0)=L \cos \theta_{0}$, with $V, \theta_{0}$ and $L$ being the striking velocity, initial yaw angle and rod length, respectively. Penetration will cease when $v(t) \leq V_{c}$ or $l_{e}(t) \leq 0$. 


\section{Figure caption list}

Fig.1 The orientation and position of the eroding rod.

Fig.2 Locations of the rod and crater sidewalls: (a) $P(t)<l_{e}(t)$ (b) $P(t) \geq l_{e}(t)$

Fig.3 Comparison of the calculated crater shape with experimental observation from [3]: (a) Predicted crater shape (b) Experimental observation [3]

Fig.4 Comparison of the predicted yaw angle with experiments and simulation results: (a) $V=1400 \mathrm{~m} / \mathrm{s}, L / d=10[9,17]$ (b) $V=1650 \mathrm{~m} / \mathrm{s}, L / d=20[18]$

Fig.5 Comparison of the predicted critical yaw angle with empirical formula [3].

Fig. 6 Variation of yaw angel during the penetration.

Fig.7 The influence of $\lambda, \varsigma$ and $\psi$ on critical yaws for a given tungsten alloy rod and steel targets.

Fig.8 Relationship between $\theta_{c 1}$ and $\theta_{c 2}$ for $\lambda=2, \psi=1 / 10$ at different striking velocities.

Fig.9 The influence of $\lambda, \varsigma$ and $\psi$ on critical yaws for steel rods and a given aluminum alloy target.

Fig.10 The influence of $\lambda, \varsigma$ and $\psi$ on critical yaws for steel rods and steel targets.

Fig.11 Comparisons of the new empirical formula with the predicted results from iterative algorithm:

(a) Tungsten alloy rod penetrating steel targets (b) Steel rods penetrating aluminum alloy target(c) Steel rods penetrating steel targets

Fig. 12 Comparisons of the new empirical formula Eq. (19) with the empirical formula proposed in Ref. [3]. 